\title{
High Performance Doa Trackers Derived From Parallel Low Resolution Detectors
}

\author{
Ana Perez-Neira, Miguel A. Lagunas*. \\ Department of Signal Theory and Communications. Universitat Politècnica de Catalunya \\ Campus Nord UPC. Edificio D-5. c/ Gran Capità, s/n. \\ 08034 BARCELONA, SPAIN \\ Phone:34-3-4016436. Fax: 34-3-4016447. \\ e-mail: anuska@gps.tsc.upc.es
}

\begin{abstract}
Traditionally, high resolution spectral Direction of Arrival (DOA) estimation has been associated with algorithms rather than with a processing scheme or architecture. Motivated by a previous work on feasible implementations of the Estimate and Maximize algorithm [1], the authors show that classical bank filter approach [see 2 and its references] can get similar, even better, performance than the most sophisticated algorithms, in terms of performance versus complexity. In fact, the practicality and robustness required for DOA trackers, both in radar and in the mobile communication scenarios to alleviate data fusion and hand-over respectively, makes evident the use of filter-bank or scanning beams for DOA tracking at the expense of resolution. The herein reported tracker enhances complexity and robustness of these schemes, achieving high resolution from the EM architecture. The result is a low complexity tracker with robustness against coherent sources and a resolution close to Singular Value Decomposition (SVD) based methods.
\end{abstract}

\section{Introduction}

Motivated by a previous work on feasible implementations of the Estimate and Maximize algorithm [1], the authors show that classical bank filter approach [2] can get similar, even better, performance than the most sophisticated trackers in terms of performance versus complexity. The present summary is organized as follows: Section 2 goes over the scanning beam procedures for DOA estimation and brings in the modifications of interest in this work. Next, Section 3 brings out the EM-based architecture in order for Section 4 to propose a multiple source tracker that uses this architecture together with the beamforming scanning approach briefly described in the previous section. The result is a DOA tracker architecture and algorithm whose robustness and performance is associated to the intrinsic clarity and simplicity of the processing scheme and the DOA algorithms used inside.

* This work has been supported by PRONTIC/CICYT: TIC-95-1022C05-01 and CIRIT/GENERALITAT de Cat. GRQ 93-3021

\section{Scanning beam procedures for Doa estimation}

In face of DOA detectors, usually based on SVD of the data matrix or its covariance, the oldest approach, referred to as the bank filter approach, uses a dedicated beam to explore all the scenario looking for the steering directions where a local maximum of received power is produced. As it can be viewed in Figure 1, the DOA estimator is implemented by a steerable beam a $\left(\mathbf{s}_{\mathrm{d}}(\theta)\right.$ denotes the steering vector, focused on angle $\theta$, to which the beam is steered) which, followed by a power device (envelope detector plus integration) produces the power density $\Phi$ (power/solid angle) for every search direction $\mathbf{s}_{\mathrm{d}}$ (the spatial bandwidth $\mathrm{BN}_{\mathrm{N}}$ is the noise bandwidth [2]). Finally, the DOA estimate will be the maximum of the spatial power density.

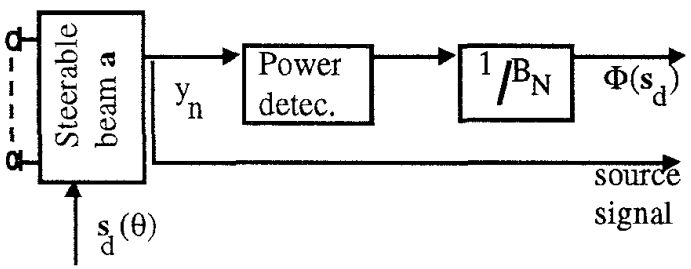

Figure 1. Scanning beam scheme for DOA estimation

From the simplicity of the scheme depicted in figure 1, it can be concluded that complexity and robustness of these procedures are their main features. It is in terms of resolution when the main criticism appears. Any DOA estimation procedure using a beamvector to measure power density has to face the uncertainty principle being the product of the aperture size in wavelenghts by the beamvector bandwidth bounded. An example is the classic phased-array scanning procedure.

The phased-array scanning (1) can be formulated as a beamformer a with $0 \mathrm{~dB}$ gain in the steered direction $\mathbf{s}_{\mathrm{d}}$ and minimizing the response to the non-directional noise (with identity covariance matrix)
$a^{H} s_{d}=1 \quad$ (1.a)
$a^{H} s_{d}=1$
$\left.a^{H} a\right|_{\min }$ (1.b)
$a^{H} \boldsymbol{R} a l_{\min }$ 
The phased-array response is distorted whenever nonuniform spatial noise or source distributions are to deal with. To alleviate in part the resulting leakage problem the so-called Capon's beamformer is designed in a data dependent fashion. The Capon's beamformer adapts to the spectral content of the input process at each DOA of interest. For each scan direction, it reduces the interference contributions to noise level. Its basic formulation is shown in (2), where $\mathbf{R}$ is the data correlation matrix measured from the snapshot vector $\mathbf{x}$.

Nevertheless, the case of interest of the hereafter tracker is that both approaches, i.e. the data independent or phased array and the data-dependent or Capon's beamvector, are suitable for introducing additional constrains. Specifically, the modification we are interested in is when a given direction $s_{0}$ has to be nulled out in order to reduce leakage in any $s_{\mathrm{d}}$ due to the potential presence of an interference source at $s_{0}$. The resulting beamvector comes from the following formulation in (3) and (4)

$$
\begin{aligned}
& a^{H}\left(s_{d} s_{O}\right)=\left(\begin{array}{llll}
l & 0
\end{array}\right)(3 a) \quad a^{H}\left(s_{d} s_{O}\right)=\left(\begin{array}{ll}
1 & 0
\end{array}\right) \\
& \left.a^{H} a\right|_{\min } \\
& \boldsymbol{a}^{H} \boldsymbol{R} \boldsymbol{a} \|_{\min }
\end{aligned}
$$

where (3) and (4) depart from the phased-array and the Capon's beamformer philosophy respectively. The corresponding beamvectors are easily derived by means of the Lagrange multipliers. For its simplicity, we pay special attention to the beamvector that is derived from (3) and formulated in (5).

$\boldsymbol{a}=\boldsymbol{A}\left[\boldsymbol{A}^{H} \boldsymbol{A}\right]^{-1}(1 ; 0)=\boldsymbol{A}^{\#}(1 ; 0)$

where $\boldsymbol{A}=\left[\begin{array}{ll}s_{d} & s_{O}\end{array}\right]$ and \# stands for the pseudo-inverse. This minimum norm beamvector leads to minimum loss of desired signal response if the coefficients $a_{i}$ are achieved by attenuation and to smallest sensitivity to errors in construction. Additionally, its design is completely data free. It is also interesting to note that if the steered direction $\mathbf{s}_{\mathrm{d}}$ is the same as the desired source direction, the beamvector formulated in (5) offers the Deterministic Maximum Likelihood estimate of the signal waveform $\hat{\mathrm{e}}$ coming from that source.

$\hat{e}=A^{\#}(1 ; 0) x$

We recall the importance of the noise bandwidth $\left(\mathrm{B}_{\mathrm{N}}\right)$ normalization in order to get a reliable DOA estimate from the spatial power density $\Phi$ in (3)instead of directly using the spatial power.

$\Phi=\boldsymbol{a}^{H} \boldsymbol{R} \boldsymbol{a} / \boldsymbol{B}_{N}$

To be more specific, spatial bandwidth may introduce substantial power leakage from sources or directional noise impinging on the aperture from other directions than the desired one.

It is important to remark the robustness and low complexity of both procedures associated with the principle of the beamvector scanning. The only problem they face is spatial frequency leakage or resolution loss for the multiple source case.
Next, we will propose to use either (3) or (4) in a EM based architecture to provide a high resolution tracker yet preserving the low complexity and robustness previously mentioned.

\section{The EM-based architecture}

After a detailed exam of the EM algorithm [1] both in the deterministic and the stochastic approach, the Estimate step can be viewed as a blocking step where the multiparameter estimation problem is reduced to a single parameter estimation. Being more specific, the steps Estimate and Maximize, when implemented in a signal processing architecture for DOA estimation, can be renamed as blocking and single source estimation respectively. In other words, given the original data snapshot $\mathbf{x}_{\mathbf{n}}$ containing NS sources, the blocking step produces NS snapshots $\mathbf{x}_{\mathrm{n}, \mathrm{k}}(\mathrm{k}=1, \mathrm{NS})$ such that a single source is relevant in every snapshot or, at least, the other sources are highly attenuated with respect to this source.

In consequence, the blocking step could be implemented as NS matrices $\mathbf{B}_{\mathbf{k}}(\mathrm{k}=1, \mathrm{NS})$ that produces from $\mathbf{x}_{\mathbf{n}}$ the single source snapshot

$$
\boldsymbol{x}_{n, k}=\boldsymbol{B}_{k} \boldsymbol{x}_{n} \quad(k=1, N S)
$$

As the blocking step requires the source DOA's, it is necessary to feed the DOA's obtained in the second stage back to the first or blocking stage. This is the other main feature of the EM and EM-based algorithms. As the reader can observe in figure 2, the maximum at the output of each branch governs the nulls of the other branches (i.e. cross-feedback). This fact prevents two or more branches from collapsing into the same angle estimation.

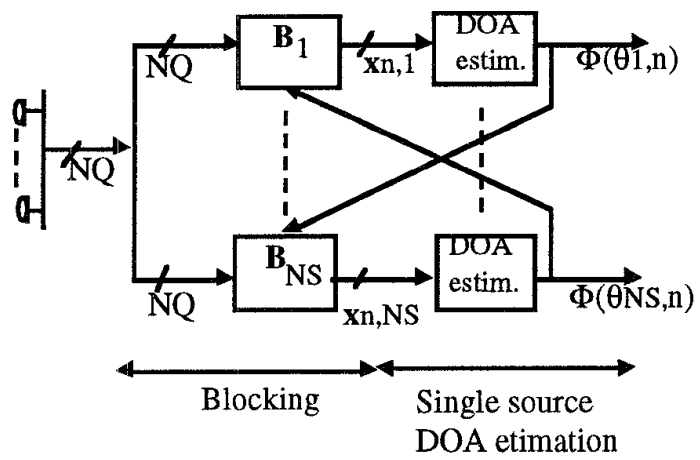

Figure 2. The EM-based architecture with the blocking stage followed by single source DOA detectors

The resulting architecture, depicted in the figure 2, can be found in detail in [3], where the links with the deterministic and stochastic EM algorithm are presented in full. The purpose of this work is to use this architecture together with the beamforming scanning approach briefly described in the previous section.

From now on, the presentation will be reduced to the two source case for a linear array. As the reader may 
conclude there is no formal difficulties to extend the application to the case of planar arrays or to the case of multiple sources. Nevertheless, in a radio communication scenario, the probability of more than two users demanding Space Diversity Multiple Access (SDMA) is very low being, in consequence, the case of two active sources the closest to real scenarios.

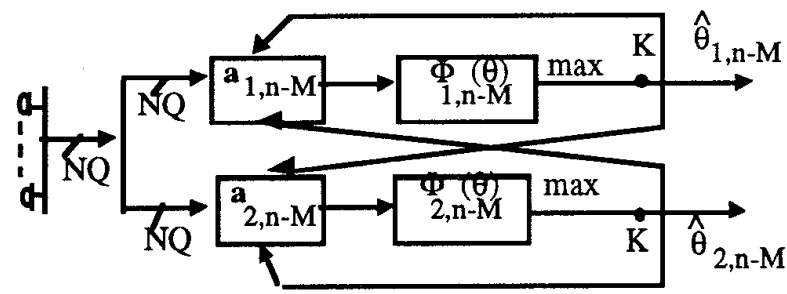

\section{Figure 3. A DOA tracker with beamformer scanning procedures in an EM-based architecture.}

Finally, and going back to the architecture of figure 2, it should be mentioned that both steps can be implemented as a single one when the procedures shown in (3) and (4) are used. These procedures allow the packing of both steps in a single one; since, being single source estimates, they include the blocking of DOAs a priori selected. This proposed architecture is the one depicted in figure 3.

Next section will explain how a two source DOA detector/tracker based in this architecture works.

\section{The proposed multiple source tracker.}

\section{IV.1. A DOA tracker architecture and algorithm}

In the EM-based architecture that is depicted in figure 3 , two source DOA's are produced: $\hat{\theta}_{1, n-M}$ and $\hat{\theta}_{2, n-M}$. Initially, the data correlation matrix $\mathbf{R}_{\mathrm{n}}$, which is required to compute the spatial power density $\Phi_{i}$, is initalized with a number of snapshots equal to ten times the number of sensors and afterwards this matrix is updated during $M$ snaphots following the rule:

$\boldsymbol{R}_{n-M+k}=\beta \boldsymbol{R}_{n-M}+(1-\beta) \boldsymbol{x}_{n-M+k} \boldsymbol{x}_{n-M+k}^{H}$

being $M$ equal to $1 / 1-\beta$. This interval $M$ is the number of samples between successive updates of the DOAs provided by the system. Its choice is a trade-off between radial source velocity and scanning time.

To make the system robust to bad initializations in whatever kind of scenario (i.e. very different power sources and even presenting strong correlation), initially, just one branch sets out to work. Once, this branch has detected one source DOA, this DOA can then drive the null of the second branch. In this way, both branches cannot collapse into the same source DOA. If both DOA's are far enough, both branches in figure 3 can then begin to scan parallelly. Next, the procedure to update each angle estimate is described.

During the mentioned $\mathbf{M}$ snapshots and in the case of the data independent design (see 3), the beamformers are obtained as it is shown in (10) for the beamforemer labelled 1 in figure 3 and in the same manner for the beamformer 2

$$
\begin{aligned}
& \stackrel{H}{a_{I n}}\left[s_{1}(\theta) \quad s_{2}\left(\hat{\theta}_{2, n}\right)\right]=\left(\begin{array}{ll}
1 & 0
\end{array}\right) \\
& \left.\underset{a_{I n}}{H} a_{1 n}\right|_{\min }
\end{aligned}
$$

Note that for the beamformer labelled 1 , the second branch drives its null at $\hat{\theta}_{2, \mathrm{n}}$ and in the same manner for the beamformer 2

Once the beamformers have been designed, they scan on $s$ as:

$$
\Phi_{\ln }(\theta)=\frac{\stackrel{H}{a_{I n}} \boldsymbol{R}_{n} \boldsymbol{a}_{\ln }}{\boldsymbol{a}_{\ln }^{H} \boldsymbol{a}_{\ln }}
$$

where the spatial bandwidth $\mathrm{BN}_{\mathrm{N}}$ has been approximated by the norm of the beamvector $\mathbf{a} 1 \mathrm{n}$ [3]; the new estimate will be the DOA that maximizes the estimated spatial power density

$$
\hat{\theta}_{l, n}=\max \Phi_{I n}(\theta)
$$

We remark that, in order to save in time and computational burden the DOA's that are scanned on $s$ can be close to the previous $\hat{\theta}_{1, n-M}$. However, the system is no more a detector but just a tracker.

Note that simultaneously to the acquisition period $\mathrm{M}$, the architecture may iterate over (10)-(12) in the same fashion as it was in the original EM algorithm. It should be pointed out that the number of iterations, to be useful, requires a high precision scanning through $\mathbf{s}$ and at the end may face the upper bound in resolving two close sources from a $\mathbf{M}$ interval data correlation estimate. At least two iterations will be necessary in any case.

\section{IV.2. Tracking subsystem}

The concept of a global tracker includes not only the DOA detection scheme, but also the parameter filtering which enables to cope with eventual fadings of bounded time duration, as it may occur in crossing radial trajectories of two targets. This additional processing uses to contain two additional stages of time-trackers of each parameter and data fusion or, in some cases, image processing of the two image produced by parameter values versus time.

Most of the cases, the DOA detection scheme (we just described an alternative in the previous section) does not take profit from the powerful processing that follows in forming the global tracker system. We comment on it to state that the blocking plus estimation scheme described can take a great advantage of the tracker subsystem. We will refer hereafter as parameter tracking, since most of the success of the DOA estimation is based on adequate 
nulling or inhibition of the non-steered sources.

Once the detected angle has stabilised at each of the branches, an elevation tracker is used in the scheme of figure 3 (insertion point $\mathrm{K}$ ). In this way, the performance of the system may improve since tracking and prediction of "next" location is of capital importance in inhibition or blocking.

This work has employed the most basic parameter tracker that can be used: a Kalman filter tracking radial position $\hat{\theta_{i e}, n-M}$ and $\hat{v_{i e}, n-M}$ velocity. A complete description can be found in [4]. Next, we just comment some specific aspects on the state equation and the measurement equation. The state model is

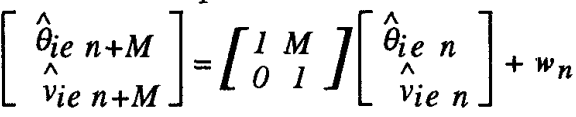

where $w_{n}$ is the uncertainty (associated to the maneuverability of the sources) with covariance matrix $\mathbf{Q}$. The measurement model is (14)

$$
\hat{\theta}_{i n}=\theta_{i e n}+v_{n}
$$

where $\hat{\theta}_{\mathrm{i} n}$ is the estimate produced after the detection and $v_{n}$ is the noise in the observation of the elevation angle. This noise, of covariance $c_{\mathrm{vn}}$, is due to air-interface, down conversion mismatching, noise and DOA detection errors.

Both covariance $\mathbf{Q}$ and $c_{\mathrm{v}}$ have to be matched to the specific application. In our work we have set $Q$ to diagonal $\left(10^{-4} 10^{-7}\right)$ for a mobile communication scenario. We have commented before that, simultaneously to the acquisition period $\mathrm{M}$, the architecture of figure 2 may iterate over (10)-(12). During these L iterations, each of the detectors adjust their DOA estimates until each one stabilizes. The, the Kalman sub-system filters the noise out of these estimates and produces $\hat{\theta}_{i e} n$. Therefore, in order to set the measurement covariance $c_{\mathrm{vn}}$, it can be estimated as $c_{v n}^{L}$ in (15), that is, the error power between the angle predicted by Kalman $\hat{\theta}_{i e} n$ and the angle detected $\hat{\theta}_{i n}$ over L realizations

$$
c_{v n}^{k+1}=(1-1 / L) \quad c_{v n}^{k}+1 / L\left|\hat{\theta}_{i n}^{k}-\hat{\theta}_{i e n}\right|^{2}
$$

$k=I . . L$

\section{Simulations and Conclusions}

In order to validate the proposed DOA detection/tracking technique two simulations have been conducted. Figure 4 and 5 show the case of two moving sources tracked by the system of figure 3 , where the beamformers $\mathbf{a}_{i}$ are simple phased-arrays that follow the design rule of (10) and where the Kalman sub-system is incorporated. First. figure 4 shows the performance of the system in a scenario of two sources received with very different powers: 15 and $5 \mathrm{~dB}$ respectively. The uniform linear array consists of 8 sensors and each scan is carried out after 30 snaphsots. Radial velocity is $0.01 \%$ snapshot and an initial angle estimate of $20^{\circ}$ has been considered.



Figure 4. Two sources of [15 5] dB. Tracking by an $B$ sensor array. Each scan consists of 30 snaphots.

Next, figure 5 is carried out in the same scenario but with fully coherent sources.

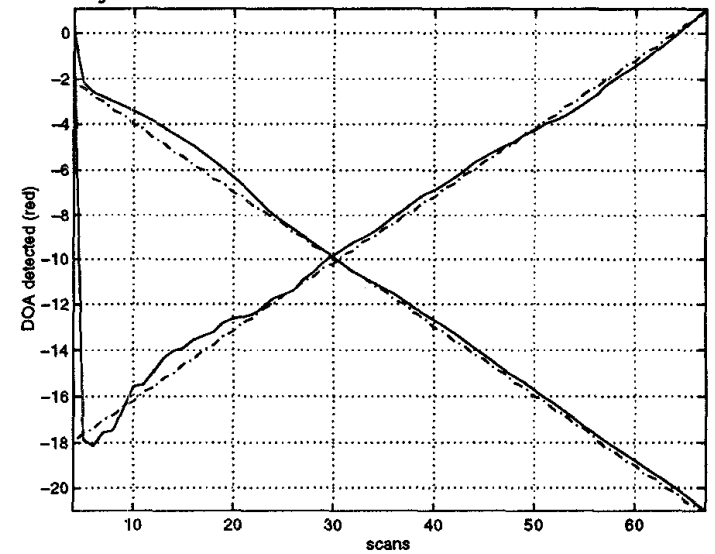

Figure 5. Two fully coherent sources of [15 5] dB. Tracking by an 8 sensor array. Each scan consists of 30 snaphots.

The proposed technique offers a good trade-off between performance against complexity and cost. Its robustness is associated to the intrinsic clarity and simplicity of the processing scheme and the DOA algorithm used inside.

Future work will consider the impact of the deviation in element locations, mutual coupling an quantization effects in using digitally controlled attenuators and shifters.

\section{References}

[1] M.Feder, E.Weinstein, "Parameter Estimation of Superimposed Signals Using the EM Algorithm," IEEE Trans. on ASSP, vol. 36, no. 4, April 1988, pp. 477489

[2] M.A.Lagunas et al., "Maximum Likelihood Filters in Spectral Estimation Problems," Signal Processing 10, pp. 19-34, 1986.

[3] A.Perez-Neira, M.A. Lagunas, L.Kirkin, "CrossCoupled DOA trackers," submitted to the Special Issue on Advanced Communications of the IEEE S.P. Trans.

[4] B.O.Anderson,J.B.Moore, Optimal filtering, PrenticeHall, Electrical Engineering Series, pp 54- 59. 